\title{
Padrões de consumo de álcool de usuários de serviços de atenção primária à saúde de um município brasileiro
}

\author{
Patterns of alcohol consumption among users \\ of primary health care services in a Brazilian city
}

Divane de Vargas ${ }^{1}$

Marina Nolli Bittencourt ${ }^{1}$

Lúcia Pereira Barroso ${ }^{2}$

\footnotetext{
${ }^{1}$ Departamento de Enfermagem MaternoInfantil e Psiquiátrica, Escola de Enfermagem, Universidade de São Paulo. Av. Dr. Enéas de Carvalho Aguiar 419, Cerqueira Cesar. 05.403-000 São Paulo SP Brasil. vargas@usp.br

${ }^{2}$ Instituto de Matemática e Estatística, Universidade de São Paulo.
}

Abstract The scope of this paper was to identify alcohol consumption patterns among users of primary health care services in the city of Bebedouro in the state of São Paulo, verifying the association between usage patterns and demographic and behavioral variables of the sample. It is a cross-sectional study involving 755 subjects over 18 years of age who sought primary health care services in the city of Bebedouro. To investigate patterns of alcohol use, the Alcohol Use Disorders Identification Test (AUDIT) was used. For statistical anal$y$ sis the chi-square and binary logistic regression tests were used. The results showed that $78 \%$ of users were abstainers or low risk users, 22\% had problems with alcohol, 10\% were risk cases, $2 \%$ were high-risk users and $10 \%$ probable addicts. The greatest predictors of usage risk were males, income > than 6 minimum wages and smokers; for addicts the most important predictors were: males, working in commerce and smokers. A significant proportion of users showed patterns of problematic use of alcohol, which reinforces the need for preventive actions, since the primary health care services are an excellent opportunity to combine opportunities for screening and usage strategies for reducing harmful effects related to the problematic use of alcohol.

Key words Alcoholism, Screening programs, Primary health care, Substance-related disorders
Resumo Este estudo objetivou identificar o padrão de consumo de álcool de usuários de serviços da Atenção Primária à Saúde (APS) da cidade de Bebedouro (SP), verificando a associação entre os padrões de uso e as variáveis sociodemográficas e comportamentais. Trata-se de um estudo transversal envolvendo 755 sujeitos maiores de 18 anos, atendidos em serviços de APS. Para a investigação dos padrões de uso do álcool utilizou-se o AUDIT. Na análise estatística empregou-se o teste qui-quadrado e a regressão logística binária. Observou-se que $78 \%$ dos usuários eram abstinentes ou faziam uso de baixo risco e $22 \%$ faziam uso problemático do álcool, dos quais 10\% uso de risco, 2\% uso nocivo e $10 \%$ provável dependência. Os maiores preditores para o uso de risco foram gênero masculino; renda > que 6 salários mínimos e tabagismo; para provável dependência, os maiores preditores foram: sexo masculino, trabalhar no comércio e tabagismo. Parcela significativa dos usuários apresentou padrões problemáticos de uso de álcool, reforçando a necessidade de ações preventivas, pois os serviços de APS são excelentes espaços para combinar oportunidades de rastreamento e utilização de estratégias para redução dos prejuízos relacionados ao uso problemático do álcool.

Palavras-chave Alcoolismo, Programas de rastreamento, Atenção Primária à Saúde, Transtornos Relacionados ao Uso de Substâncias 


\section{Introdução}

O consumo abusivo de álcool está diretamente associado a efeitos deletérios sobre a saúde do indivíduo, bem como em suas relações e posições sociais, o que o caracteriza como um dos maiores problemas de saúde pública na atualidade ${ }^{1}$. O uso crônico de álcool, a longo prazo, pode afetar todos os sistemas orgânicos do corpo, e o alcoolismo está associado a uma série de doenças e distúrbios, tais como cirrose relacionada ao álcool, pancreatite, cardiomiopatia, hemorragia digestiva, intoxicações, câncer, demência alcoólica, epilepsia, polineuropatia, depressão e síndrome alcoólica fetal ${ }^{2,3}$. No que tange aos aspectos sociais e interpessoais, o consumo dessa substância é fator contribuinte para acidentes, suicídios, homicídios, assaltos, abuso infantil, violência doméstica, conflitos conjugais, divórcio e perda de emprego, dentre outras ${ }^{2,3}$.

Conforme o último levantamento da Organização Mundial de Saúde publicado em 2011², relacionado ao status global do consumo de álcool e suas consequências para a saúde, aproximadamente $4 \%$ de todas as mortes no mundo são atribuídas ao álcool. Apesar da grande variação de consumo entre os diversos países, em 2005 o consumo foi em média 6,13 litros de álcool puro consumido por adulto em todo o mundo. Em praticamente todos os países do Continente Americano, o consumo de álcool permaneceu estável nos anos de 2001 a 2005, com um aumento de apenas 5,3\% no consumo ${ }^{2}$. No Brasil, num dos últimos estudos epidemiológicos realizados observou-se que $74,6 \%$ das pessoas entre 12 e 60 anos haviam feito o uso de álcool alguma vez na vida e que $12,3 \%$ da população brasileira preenchia critérios para dependência alcoólica ${ }^{4}$. Outro inquérito realizado em 2007 no país constatou que a frequência do consumo abusivo de bebidas alcoólicas foi de $18,0 \%$ nos últimos trinta dias anteriores à entrevista, sendo quase três vezes maior em homens do que entre as mulheres ${ }^{5}$.

Esses percentuais têm repercutido no aumento de indivíduos com problemas relacionados ao álcool nos serviços de saúde, dentre outros. Existem evidências de que $20 \%$ das pessoas atendidas em serviços de atenção primária de países industrializados faz uso excessivo do álcool ${ }^{6}$. Estudos realizados em países europeus para identificar o padrão de uso de álcool de usuários de serviços de atenção primária à saúde (APS) encontraram prevalências entre 15 e 17\% na Itália ${ }^{7}$, $8 \%$ na França ${ }^{6}$ e 20\% no Reino Unido ${ }^{8}$. Alguns estudos realizados no Brasil têm apontado prevalências significativas de usuários com problemas relacionados ao uso e abuso de álcool em serviços de saúde ${ }^{9-11}$ e, apesar dos percentuais observados serem menores do que aqueles encontrados na população geral, constituem-se num problema ainda pouco explorado no país. De um modo geral, existem evidências na literatura internacional de prevalência significativa de usuários com problemas relacionados ao álcool em serviços de atenção primária à saúde. No Brasil, evidencia-se uma escassez de pesquisas sobre esse fenômeno e nos poucos estudos realizados no país ${ }^{9-11}$ observou-se uma prevalência entre 4 e $9 \%$ de pessoas com problemas relacionados ao álcool nos serviços de atenção primária à saúde.

Os resultados apresentados neste estudo são produto de uma pesquisa mais ampla "Detecção de indivíduos com transtornos relacionados ao uso e ou abuso de álcool em serviços de Atenção Básica de Saúde de um município paulista". Parte das observações desta investigação foi divulgada em estudo prévio ${ }^{12}$, o qual focou especificamente a prevalência de dependência alcoólica entre os participantes da pesquisa. No presente estudo, os autores propõem-se a apresentar um olhar mais amplo, identificando e analisando os padrões de uso do álcool da amostra investigada. O conhecimento destes por populações diversas torna o tema relevante, principalmente porque, segundo apontamentos recentes da $\mathrm{OMS}^{2}$, o uso de risco do álcool tem repercutido em problemas sociais e de saúde tão ou mais preocupantes que a própria dependência do álcool.

Assim, considera-se que a maioria dos estudos disponíveis sobre os padrões de consumo de álcool em serviços de atenção primária provém de países desenvolvidos e constata-se que pouco se conhece sobre estes padrões em países em desenvolvimento, incluindo o Brasil. Como os resultados dos estudos realizados no país ${ }^{9-11}$ encontraram uma parcela significativa de usuários de Atenção Primária à Saúde com problemas relacionados ao beber, é importante conhecer os padrões de uso do álcool de populações diversas com vistas, dentre outros, à proposição de estratégias preventivas. O presente estudo objetivou identificar o padrão de consumo de álcool de usuários de serviços da Atenção Primária à Saúde da cidade de Bebedouro, São Paulo, verificando a associação entre os padrões de uso e as variáveis sociodemográficas e comportamentais da amostra. 


\section{Métodos}

Estudo transversal realizado na cidade de Bebedouro, situada no nordeste do estado de São Paulo, com uma população estimada em 77.626 habitantes em 2009. Os serviços públicos de saúde do município constituem-se atualmente por um hospital geral, dezesseis Unidades Básicas de Saúde (UBS), 11 equipes de Estratégia Saúde da Família (ESF) e dois Centros de Atenção Psicossocial (CAPS). $\mathrm{O}$ atendimento médico realizado pelo Sistema Único de Saúde (SUS) está estimado numa média de $3,3 \%$ para cada 1.000 habitantes.

Os dados foram coletados nos quatro conglomerados que agregavam a Unidade Básica de Saúde (UBS) e a Unidade de Saúde da Família (USF). A amostra constituiu-se de 755 sujeitos maiores de dezoito anos, de ambos os sexos, que procuraram atendimento nos locais de coleta, independente do motivo, sendo então recrutados segundo critérios de conveniência. Para coleta dos dados foram treinados 10 entrevistadores que permaneceram nos serviços envolvidos durante quatro semanas (segunda a sexta-feira), e a mesma se deu na sala de espera de cada um dos locais de coleta, o preenchimento do instrumento não ultrapassou 10 minutos.

$\mathrm{O}$ instrumento utilizado para coleta dos dados foi o AUDIT (Alcohol Use Disorders Identification Test). Trata-se de um screening com reconhecimento mundial, desenvolvido pela Organização Mundial de Saúde (OMS) na década de 1980, com objetivo de identificar bebedores de risco no ambulatório geral, medindo consumo, sintomas de dependência e consequências pessoais e sociais do beber. O teste aborda o padrão de consumo e suas consequências nos últimos doze meses, através de 10 itens, sendo três sobre o uso de álcool, quatro sobre dependência e três sobre problemas decorrentes do consumo ${ }^{13}$. As alternativas de respostas vão de 1 a 4 , sendo que pontuações altas são indicativas de problemas, essas pontuações permitem classificar o usuário em uma de quatro zonas de risco, de acordo com o escore total obtido: zona I (até 7 pontos: indica uso de baixo risco ou abstinência); zona II (de 8 a 15 pontos: indica uso de risco); zona III (de 16 a 19 pontos: sugere uso nocivo) e zona IV (acima de 20 pontos: sugestivo de dependência). Estudos brasileiros utilizando o AUDIT ${ }^{14}$ apontaram sensibilidade de $87,8 \%$ e especificidade de $81 \%$ sendo o mesmo considerado satisfatório para estudos em serviços de APS. Precedeu a aplicação do AUDIT um questionário sociodemográfico que continha também três questões referentes ao uso de tabaco, à presença de patologias crônicas e ou aquelas que poderiam estar relacionadas ao alcoolismo como úlceras e gastrites. Assim, as variáveis independentes investigadas foram sexo, idade, raça, religião, renda familiar, escolaridade, estado civil, tabagismo e a presença de patologias crônicas e ou relacionadas ao uso nocivo e à dependência alcoólica.

Para verificar o padrão de uso de álcool na amostra do estudo inicialmente verificou-se a distribuição dos usuários em cada uma das quatro zonas de classificação do AUDIT. As associações entre os padrões do uso de álcool e cada uma das variáveis investigadas no estudo foram exploradas pelo Teste de Qui-quadrado ou Teste exato de Fisher. Na sequência, buscando identificar a força das possíveis variáveis explicativas para predizer cada padrão de uso do álcool, dois modelos de regressão logística foram ajustados, um para predizer a abstinência ou uso de baixo risco versus uso de risco do álcool e outro para predizer possível dependência do álcool versus abstinência ou uso de baixo risco. Beber de risco foi considerado pontuar na Zona II do AUDIT, possível dependência pontuar na Zona IV e abstinência ou uso de baixo risco pontuar na Zona I. Para este modelo, devido ao número reduzido de indivíduos que pontuaram na Zona III do AUDIT, os mesmos foram excluídos e por isso a análise de regressão logística foi ajustada separadamente para as Zonas II e IV. Assim, a análise da associação das variáveis do estudo nas zonas II e IV tomou como referência o grupo que pontuou na Zona I. O nível de significância adotado foi de $5 \%$. As estatísticas com valor-p menor ou igual 0,05 foram consideradas significantes. Todas as análises estatísticas foram realizadas no programa computacional MINITAB $^{\circledR}$.

Os procedimentos éticos do estudo são representados pela aprovação do projeto pelo Comitê de Ética em Pesquisa com Seres Humanos das Faculdades Integradas Fafibe e pela assinatura do termo de consentimento livre esclarecido pelos sujeitos que aceitaram participar da pesquisa.

\section{Resultados}

A amostra do estudo constitui-se predominantemente de sujeitos do sexo feminino, casados, brancos, católicos, com ensino fundamental e renda familiar entre um e cinco salários mínimos (Tabela 1). A maioria (52\%) procurou o serviço buscando atendimento na clínica geral. Com relação à profissão ou ocupação, a amos- 
Tabela 1. Distribuição de risco de uso de álcool, de acordo com o AUDIT - (n = 755), dados expressos em porcentagem. São Paulo, SP, 2012.

\begin{tabular}{|c|c|c|c|c|c|}
\hline Zonas de Risco & $\begin{array}{c}\text { I } \\
(0-7 \text { pontos })\end{array}$ & $\begin{array}{c}\text { II } \\
(8-15 \text { pontos })\end{array}$ & $\begin{array}{c}\text { III } \\
\text { (16-19 pontos) }\end{array}$ & $\begin{array}{c}\text { IV } \\
(+20 \text { pontos })\end{array}$ & Total \\
\hline Total & 78 & 10 & 2 & 10 & 100 \\
\hline \multicolumn{6}{|l|}{ Gênero $(\mathrm{n}=755)$} \\
\hline Masculino & 25 & 5 & 1 & 7 & 38 \\
\hline Feminino & 54 & 4 & 1 & 3 & 62 \\
\hline \multicolumn{6}{|l|}{ Faixa etária $(\mathrm{n}=755)$} \\
\hline$<20$ & 7 & 0 & 0 & 0 & 7 \\
\hline $20-29$ & 16 & 2,6 & 0 & 2,4 & 21 \\
\hline $30-39$ & 16 & 2,3 & 0,5 & 2,4 & 21,2 \\
\hline $40-49$ & 17 & 3 & 0 & 2,0 & 22,1 \\
\hline $50-59$ & 12 & 1 & 0,4 & 1,3 & 14,7 \\
\hline$>59$ & 11 & 0,8 & 0,5 & 1,7 & 14 \\
\hline \multicolumn{6}{|l|}{ Escolaridade $(\mathrm{n}=755)$} \\
\hline Fundamental & 50,5 & 5,5 & 2 & 6 & 64 \\
\hline Médio & 23,7 & 3,2 & 0,5 & 2,6 & 30 \\
\hline Superior & 4,5 & 1 & 0 & 0,5 & 6 \\
\hline \multicolumn{6}{|l|}{ Estado civil $(\mathrm{n}=755)$} \\
\hline Casado/Amasiado & 52,3 & 6,2 & 1,5 & 5,4 & 65,4 \\
\hline Solteiro & 15,3 & 2 & 0,4 & 2,8 & 20,5 \\
\hline Viúvo & 5,2 & 0,3 & 0,3 & 0,2 & 6,1 \\
\hline Outro & 5,4 & 1,3 & 0,2 & 1,1 & 8,0 \\
\hline \multicolumn{6}{|l|}{ Renda mensal $(\mathrm{n}=755)$} \\
\hline$<1$ Sal Min & 25 & 1,2 & 1 & 4,5 & 30,1 \\
\hline 1-5 Sal Min & 50 & 7,5 & 2 & 5 & 64,5 \\
\hline 6-10 Sal Min & 2,8 & 0,9 & 0,7 & 1 & 5,4 \\
\hline \multicolumn{6}{|l|}{ Religião $(\mathrm{n}=755)$} \\
\hline Católico & 48 & 7,5 & 1,3 & 6,3 & 63,1 \\
\hline Espírita & 2,4 & 0,3 & 0,1 & 0,3 & 3,1 \\
\hline Evangélico & 24 & 1 & 0,3 & 1,5 & 26,8 \\
\hline Outros & 4,5 & 0,8 & 0 & 1,7 & 7 \\
\hline \multicolumn{6}{|l|}{ Tabagismo $(\mathrm{n}=755)$} \\
\hline Não & 63,3 & 5 & 1 & 3,2 & 73 \\
\hline Sim & 15 & 4,5 & 1 & 6,6 & 27 \\
\hline
\end{tabular}

tra constitui-se predominantemente por donas de casa (29\%) e por indivíduos que no momento da coleta desempenhavam cargos operacionais, compreendidos dentre outros, por balconistas, frentistas e vendedores (36\%). Quanto às características clínicas, 55\% dos entrevistados negou a presença de patologias crônicas ou que pudessem estar associadas com o uso do álcool, seguidos de $21 \%$ que relataram serem portadores de hipertensão arterial. Com relação à cor autorreferida, as pessoas brancas constituíram a maioria da amostra, com 63\%.

Quanto ao padrão do uso de álcool da amostra selecionada de acordo com os escores obtidos no AUDIT, observou-se que 78\% dos entrevista- dos foram classificadas na zona I, $10 \%$ na zona II, $2 \%$ na zona III e $10 \%$ na zona IV (Tabela 1 ).

A maior prevalência de abstinência ou uso de baixo risco (Zona I do AUDIT) foi encontrada em indivíduos do sexo feminino, com ensino fundamental, casados ou amasiados, na faixa etária entre 20 e 39 anos (32\%), católicos com rendimentos entre 1 e 5 salários mínimos, donas de casa, sem presença de patologias crônicas, não tabagistas e que procuraram o serviço buscando atendimento com o clínico geral (Tabela 1).

Utilizando a amostra do estudo para estimar a prevalência de indivíduos que fazem uso de risco do álcool, ou seja, participantes que pontuaram na Zona II do AUDIT, observou-se que dos 
$10 \%$ destes entrevistados, a maioria estava na faixa etária entre 20 e 49 anos, com ensino fundamental, casados e ou amasiados, com renda familiar entre 1 e 5 salários mínimos, católicos, trabalhadores do comércio ou exercendo outras funções no momento da coleta, sem queixas de doenças crônicas e que procuraram o serviços de saúde buscando atendimento com o clínico geral. Com relação ao gênero observou-se que não existiu diferença significativa entre os dois grupos já que a porcentagem de homens (5\%) é bastante próxima da de mulheres que pontuaram nessa zona de risco do AUDIT (4\%) (Tabela 1).

O perfil dos outros $10 \%$ da amostra que pontuou na Zona IV do AUDIT, escores sugestivos de dependência alcoólica, é o mesmo observado entre os indivíduos que pontuaram na Zona II, exceto para o predomínio de indivíduos do sexo masculino, aposentados e desempregados, hipertensos e em sua grande maioria, tabagistas (Tabela 1).

A associação entre as variáveis do estudo e o padrão de uso nocivo do álcool (Zona II do AUDIT) verificada por meio da regressão logística binária evidenciou associação significativa entre esse padrão de consumo de álcool e as variáveis: idade, sexo, profissão, renda, religião e tabagismo (Tabela 2). Os maiores preditores dessa associação foram encontrados nas categorias de idade entre 20 e 30 anos, exercer a profissão de motorista, ter renda familiar acima de seis salários mínimos e ser tabagista (Tabela 2).

Tabela 2. Regressão logística, análise da relação entre a classificação na Zona II do AUDIT (Uso de risco) com as variáveis significativas no estudo. São Paulo, Brasil, 2012.

\begin{tabular}{lccc}
\hline \multicolumn{1}{c}{ Variáveis } & OR & IC 95\% & P \\
\hline $\begin{array}{l}\text { Idade } \\
\text { 20 a 29 anos }\end{array}$ & 3,63 & $1,61-8,19$ & 0,002 \\
30 a 39 anos & 2,25 & $1,00-5,06$ & 0,049 \\
$\quad 40$ a 49 anos & 2,73 & $1,28-5,85$ & 0,010 \\
Sexo & & & \\
$\quad$ Masculino & 2,36 & $1,34-4,16$ & 0,003 \\
$\begin{array}{l}\text { Profissão } \\
\quad \text { Motorista }\end{array}$ & 3,03 & $1,00-9,16$ & 0,050 \\
$\quad \begin{array}{l}\text { Renda } \\
\quad 1 \text { a 5 SM }\end{array}$ & 2,50 & $1,17-5,35$ & 0,018 \\
$\quad$ 6 SM & 4,88 & $1,48-6,05$ & 0,009 \\
Religião & & & \\
$\quad$ Católica & 2,06 & $1,12-3,80$ & 0,020 \\
Tabagismo & & & \\
$\quad$ Sim & 3,38 & $1,96-5,83$ & 0,000 \\
\hline
\end{tabular}

Quando se realizou a regressão logística considerando o grupo de indivíduos com possível dependência de álcool (Zona IV do AUDIT) e as variáveis envolvidas no estudo, observou-se que as variáveis: idade, sexo, profissão, estado civil e tabagismo, associaram-se positivamente a esse padrão de consumo (Tabela 3). Os maiores preditores dessa associação foram encontrados para as categorias de sexo masculino, trabalhar no comércio, estar desempregado e ser tabagista. Além disso, observou-se também que um indivíduo casado ser classificado na Zona IV do AUDIT é 2,42 vezes a chance de um indivíduo que informou outro estado civil ser classificado nessa zona.

\section{Discussão}

Os resultados deste estudo indicaram que aproximadamente $22 \%$ dos usuários de atenção primária à saúde entrevistados apresentaram, segundo os pontos de corte do AUDIT, uso problemático de álcool (uso de risco, uso nocivo e provável dependência). No que se refere a esta última, os resultados observados são semelhantes aos índices nacionais. Estimou-se em 2005 que a prevalência de dependência alcoólica na população brasileira era de $12,3 \%{ }^{3}$, resultado semelhante ao observado neste estudo em que a prevalência verificada foi de $10 \%$. Esta variação pode estar relacionada tanto ao critério amostral, quanto às próprias características dos sujeitos

Tabela 3. Regressão logística, análise da relação entre a classificação na Zona IV do AUDIT (Dependência alcoólica) com as variáveis significativas no estudo. São Paulo, Brasil, 2012.

\begin{tabular}{lccc}
\hline \multicolumn{1}{c}{ Variáveis } & OR & IC 95\% & P \\
\hline Idade & & & \\
$\quad 20$ a 29 anos & 2,89 & $1,03-8,05$ & 0,043 \\
30 a 39 anos & 3,36 & $1,41-8,05$ & 0,006 \\
$\quad 40$ a 49 anos & 2,67 & $1,10-6,47$ & 0,029 \\
$\begin{array}{l}\text { Sexo } \\
\quad \text { Masculino }\end{array}$ & 5,25 & $2,78-9,94$ & $<0,001$ \\
Profissão & & & \\
$\quad$ Comércio & 8,71 & $2,02-7,52$ & 0,004 \\
$\quad \begin{array}{l}\text { Desempregado } \\
\text { Aposentado }\end{array}$ & 4,25 & $1,54-11,68$ & 0,005 \\
$\quad 2,94$ & $1,11-7,82$ & 0,030 \\
Estado civil & & & \\
$\quad$ Casado & 2,42 & $1,23-1,78$ & 0,006 \\
Tabagismo & & & \\
$\quad$ Sim & 7,16 & $3,93-13,03$ & $<0,001$ \\
$\quad$ & & & \\
\end{tabular}


recrutados em serviços de atenção primária, ao passo que os estudos nacionais configuram-se como estudos domiciliares. Esse resultado é corroborado por estudos ${ }^{15}$ que têm apontado uma prevalência de dependência de álcool entre 6 e $10 \%$ na população brasileira, existindo uma variação entre 1 a $10 \%$ do grupo caracterizado como alcoolistas ou "grandes bebedores". Quando comparados os resultados desta pesquisa com os obtidos em estudos já realizados nos serviços de APS no Brasil ${ }^{9-11}$ observa-se que a prevalência de usuários com padrão de uso problemático do álcool é maior, no entanto, é semelhante à prevalência observada nos estudos internacionais ${ }^{6-8}$.

Com relação ao perfil dos indivíduos que apresentaram pontuações sugestivas de problemas relacionados ao álcool no AUDIT, observase que em todos os padrões de uso problemático (uso de risco, uso nocivo e provável dependência), o perfil se assemelha, constituindo-se basicamente por homens com renda familiar entre $1 \mathrm{e}$ 5 salários mínimos, na faixa etária entre os 20 e 49 anos. Esse resultado sugere que aqueles indivíduos que iniciam com o uso prejudicial do álcool, têm chances de desenvolver a dependência. Este resultado é corroborado pelos apontamentos do II Levantamento Domiciliar realizado no país ${ }^{4}$, que mostrou que de cada quatro pessoas do sexo masculino que fazem uso de álcool na vida no Brasil, uma se tornará um dependente, para o sexo feminino essa proporção era de dez para uma.

Um dos fatores que pode contribuir para esse fenômeno está relacionado, dentre outros, ao fato de esses bebedores não terem recebido informações devidas, ao longo do tempo, sobre seu padrão de uso do álcool o que contribui para a evolução desse padrão de consumo. Daí a importância de utilizar estratégias de rastreamento e intervenção junto a indivíduos com uso problemático do álcool em serviços de saúde, especialmente naqueles de atenção primária, já que estes constituem-se na principal porta de entrada dessas pessoas no sistema de saúde do país.

Consistente com a maioria das pesquisas sobre o uso de álcool, no que se refere ao gênero, neste estudo observou-se que os homens tiveram um padrão de beber em maior quantidade que mulheres em todos os tipos de uso, exceto no padrão de uso de risco (Zona II do AUDIT), nessa zona observou-se que ambos mostraram padrões semelhantes de uso, as diferenças de gênero no consumo de álcool são fundamentadas de modo que cada cultura distingue os papéis a serem desempenhados socialmente. Assim, à medida que os papeis da mulher tem se tornado mais similares aos do homem, o mesmo ocorre com seus padrões de uso do álcool ${ }^{16}$. Já no que se refere ao uso pesado ou sugestivo de dependência alcoólica, os resultados da pesquisa são consistentes com levantamentos anteriores ${ }^{4-10}$, apontando o predomínio de indivíduos do sexo masculino nesse padrão de uso, pois a proporção de indivíduos classificados na Zona IV do AUDIT (possível dependência) foi de 2,5 homens para cada mulher, resultado semelhante ao encontrado no último levantamento realizado no país, que apontou uma proporção de uma mulher para cada três homens dependentes do álcool.

$\mathrm{Na}$ análise das possíveis variáveis explicativas para predizer o uso de risco e a dependência do álcool observou-se que os maiores preditores comuns aos dois tipos de uso foram encontrados para o sexo masculino, com idade entre 20 e 49 anos, e tabagistas. Este resultado é consistente com pesquisas anteriores que também constataram uma prevalência de uso regular e dependência alcoólica maior entre os homens ${ }^{9-11}$, desemprega$\operatorname{dos}^{17}$ e tabagistas ${ }^{13,15,17}$. Observou-se que o desemprego, constituiu-se como uma categoria explicativa para predizer a dependência de álcool, a esse respeito a Organização Mundial da Saúde ${ }^{2}$ tem apontado a ligação entre uso abusivo de álcool e desemprego, com uma relação causal sendo estabelecida em ambos os sentidos, ou seja, com o uso abusivo de bebidas alcoólicas levando ao desemprego e com a perda de trabalho resultando em consumo abusivo de bebidas alcoólicas.

Por outro lado, o estresse laboral também tem sido apontado pelos estudiosos como uma das causas do uso deletério de álcool. Estudo realizado nos Estados Unidos em uma amostra de aposentados ${ }^{18}$ constatou que o consumo de álcool não sofreu alteração mesmo com a retirada dos fatores estressantes no trabalho, os autores do estudo apontaram como uma possível explicação para esse comportamento o fato de a aposentadoria contribuir para a retirada dos controles sociais existentes no ambiente de trabalho, os quais "brecariam" o uso de álcool. Ou seja, este como "automedicação" para o estresse do ambiente de trabalho sofre modulação pelas normas sociais desse contexto e que com a chegada da aposentadoria e com o fim da imposição desses "controles", esse uso de álcool tende a persistir.

Esses dados corroboram os resultados deste estudo, o qual evidenciou que o fato de o indivíduo ser aposentado constitui-se numa das possíveis variáveis explicativas para predizer a dependência do álcool na população estudada. Esse resultado é consistente com os de um estudo bra- 
sileiro ${ }^{19}$ realizado para avaliar a qualidade de vida de uma amostra de indivíduos aposentados que identificou uma prevalência de 5,7\% de dependentes de álcool na amostra estudada, relatando inclusive a necessidade de excluir da amostra sujeitos incapacitados de responder ao instrumento devido ao alcoolismo.

A profissão de motorista foi apontada neste estudo como um preditor para o uso de risco do álcool. Estudo ${ }^{20}$ realizado com mais de 1.000 sujeitos, composto em sua maioria por indivíduos que desempenhavam a profissão de motorista, utilizando o mesmo questionário desta pesquisa, evidenciou que pelo menos $40 \%$ da amostra fazia uso problemático do álcool, e desses 3,6\% preenchiam critérios de provável dependência. De acordo com o estudo ${ }^{20}$, apesar da gravidade desse problema e de sua magnitude o Brasil se encontra em fase inicial de reconhecer o comportamento do motorista com problemas relacionados ao álcool como um grave problema de saúde pública e que as poucas estatísticas apontam um quadro preocupante.

A idade foi apontada nesta pesquisa como uma possível variável explicativa tanto para predizer o uso de risco do álcool quanto a provável dependência, observou-se ainda que idade e consumo de risco do álcool tendem a ser inversamente proporcionais, ou seja, quanto mais jovem maior a chance de enquadrar-se nesse tipo de uso. Observou-se que um dos maiores preditores do uso de risco do álcool foi estar na faixa etária entre 20 e 29 anos, dado também encontrado em pesquisa prévia ${ }^{21}$, enquanto que ter entre 30 e 39 anos constituiu-se um dos maiores preditores para ser classificado no tipo de uso sugestivo de dependência. Esses resultados são corroborados por estudos prévios que evidenciaram intervalos de faixa etária semelhantes entre pessoas com problemas relacionados ao álcool ${ }^{9-}$ 11,16,22. Observa-se também que esse resultado é semelhante àquele evidenciado pelo II Levantamento Domiciliar do país ${ }^{4}$ o qual encontrou que as faixas etárias com maiores porcentagens de dependentes de álcool foi de 18 a 24 anos e de 25 e 34 anos. Ainda a esse respeito, segundo o I Levantamento sobre os padrões e uso de álcool pela população brasileira ${ }^{22}$, os indivíduos mais jovens tenderam a relatar maior padrão de uso do álcool em todos os tipos de uso, observando-se que entre os maiores de 59 anos há maior abstinência, fato também evidenciado nesta pesquisa.

Segundo o I Levantamento sobre os padrões de consumo do álcool na população brasileira, a abstinência é mais de 79\% maior entre os brasileiros de 60 anos ou mais do que entre os jovens. Para os autores do levantamento ${ }^{22}$, esse resultado não surpreende já que os mais velhos tendem a ter comportamento mais conservador que os jovens e, portanto, a população mais jovem também apresenta mais problemas do que os mais velhos com relação ao beber. Por outro lado há que se considerar também que esse conservadorismo pode influenciar de forma negativa a respostas a questionários de detecção de problemas relacionados ao álcool pelos indivíduos mais velhos, levando-os a omitir seus reais padrões de uso e mostrando-se menos acessíveis a exames mais detalhados ${ }^{8}$ o que contribuiria para subestimar a prevalência de uso de álcool nessa população, já que estudos específicos ${ }^{18,19}$ têm apontado prevalência significativa de consumo problemático do álcool entre idosos.

As possíveis limitações desta pesquisa podem ser representadas pela não sistematização do grupo amostral (amostra de conveniência) e pelo reduzido período de coleta de dados (quatro semanas) em um único município brasileiro, aliada à falta de uma avaliação mais consistente para confirmação da eficácia da metodologia de deteç̧ão, o que não permite a generalização dos resultados. Por outro lado, os resultados possibilitam identificar a importância de estudos dessa natureza nos serviços de saúde, já que identificou-se um número considerável de usuários de serviços de atenção primária à saúde do município estudado que apresentaram problemas relativos ao uso do álcool, não restritos somente a dependência mas também ao uso de risco o qual tem contribuído para uma vasta gama de problemas à saúde da população. Além disso, os resultados desta pesquisa contribuem para o mapeamento dos padroes de uso do álcool em regiões do país ainda não estudadas, o que diante da escassez de dados nacionais sobre usuários de serviços de atenção primária à saúde o torna relevante. Oferecer novos subsídios para a construção do conhecimento sobre essa problemática no âmbito da saúde pública favorece a identificação da necessidade de ações de detecção e prevenção do uso problemático de álcool por usuários do sistema público de saúde do país, já que é possível que essa situação seja comum também em outras regiões brasileiras, sendo desejável inclusive que sejam desenvolvidas outras pesquisas dessa natureza em outros locais e utilizando-se de outros instrumentos e técnicas. 


\section{Conclusão}

Parcela significativa dos usuários dos serviços de atenção primária estudada apresentou padrão problemático de uso de álcool. O estudo evidenciou que as variáveis: gênero, idade e tabagismo são possíveis preditoras desse consumo (uso de risco, uso nocivo e possível dependência). Homens com idade entre 20 e 49 anos e tabagistas, compõem um grupo com maior risco para o uso problemático do álcool e, portanto, mais suscetíveis à morbidade e mortalidade relacionadas a esse uso. O perfil dos indivíduos que foram classificados em zonas indicativas de problemas relacionados ao álcool é semelhante, o que indica que existe uma evolução do padrão do uso do álcool dada a não detecção e informação dos mesmos em seus estágios iniciais, o que reforça a necessidade de ações de cunho preventivo nesses cenários, principalmente porque os serviços de atenção primária à saúde constituem-se num excelente espaço para combinar oportunidades de rastreamento e utilização de estratégias para redução dos prejuízos relacionados ao uso problemático do álcool.

\section{Colaboradores}

D Vargas participou da concepção do estudo, revisão da literatura, coleta e análise dos dados, redação e revisão do manuscrito. LP Barroso participou do tratamento estatístico dos dados, discussão dos dados, redação e revisão do manuscrito. MN Bittencourt participou revisão da literatura, redação e revisão final do manuscrito. 


\section{Referências}

1. Brasil. Ministério da Saúde (MS). Secretaria de Atenção a Saúde. SVS/CN-DST/AIDS. A Política do Ministério da Saúde para Atenção Integral a Usuários de Álcool e outras Drogas. Brasília: MS; 2011. [site da Internet]. [acessado 2013 out 11]. Disponível em: http://bvsms.saude.gov.br/bvs/publicacoes/pns_ alcool_drogas.pdf.

2. World Health Organization (WHO). WHO Global Status Report on Alcohol 2011. Management of substance abuse: alcohol.[site da Internet]. [acessado 2013 out 13]. Disponível em: http://www.who.int/ substance_abuse/publications/alcohol/en/

3. Brasil. Secretaria Nacional de Políticas sobre Drogas. Relatório brasileiro sobre drogas. Brasília: SENAD; 2009. [site da Internet]. [acessado 2013 out 13]. Disponível em: http://www.obid.senad.gov.br/ portais/OBID/biblioteca/documentos/Relatorios/ 328379.pdf

4. Carlini EA, Galduroz JCF. II Levantamento domiciliar sobre o uso de drogas psicotrópicas no Brasil: estudo envolvendo as 108 maiores cidades do país. [site da Internet]. [acessado 2013 out 13]. Disponível em: http://www.obid.senad.gov.br.

5. Brasil. Ministério da Saúde (MS). Secretaria de Vigilância em Saúde. Secretaria de Gestão Estratégica e Participativa. Vigitel Brasil 2010: Vigilância de fatores de risco e proteção para doenças crônicas por inquérito telefônico. Brasília: MS; 2011.

6. Gache P, Michaud P, Landry U, Accietto C, Arfaoui S, Wenger O, Daeppen JB. The Alcohol Use Disorders Identification Test (AUDIT) as a Screening Tool for Excessive Drinking in Primary Care: Reliability and Validity of a French Version. Alcohol Clin Exp Res 2005; (29)11:2001-2007.

7. Agabio R, Nioi M, Serra C, Valle P, Gessa GL. Alcohol use disorders in primary care patients in $\mathrm{Ca}$ gliari, Italy. Alcohol Alcohol 2006; 41(3):341-344.

8. Coulton S, Drummond C, James D, Godfrey C, Bland JM, Parrott S, Peters T. Opportunistic screening for alcohol use disorders in primary care: comparative study. BMJ 2006; 4(51):1-7.

9. Ronzani TM. Padrão de uso de álcool entre pacientes da atenção primária à saúde: estudo comparativo. Rev. APS 2008; (11)2:163-171.

10. Magnabosco MB, Formigoni MLOS, Ronzani TM. Avaliação dos padrões de uso de álcool em usuários de serviços de Atenção Primária à Saúde de Juiz de Fora e Rio Pomba (MG). Rev. Bras. Epidemiol. 2008; 10(4):637-647.

11. Bortolozzi MC, Traebert J, Loguercio A, Kehrig R. Prevalência e perfil dos usuários de álcool de população adulta em cidade do sul do Brasil. Cien Saude Colet 2010; 15(3):679-685.
12. Vargas D, Oliveira MAF, Araujo EC. Prevalência de dependência alcoólica em serviços de atenção primária à saúde de Bebedouro, São Paulo, Brasil. Cad Saude Publica 2009; 25(8):1711-1720.

13. Barbor TF, La Fuente Junior, Saunders JB, Grant M, AUDIT. The alcohol use disorders identification test: guidelines for use in primary health care. WHO Publication 1992; 94(4):1-29.

14. Mendéz EB. Uma Versão Brasileira do AUDIT (Alcohol Use Disorders Identification Test) [dissertação]. Pelotas (RS): Universidade Federal de Pelotas; 1999.

15. Galduroz JCF, Caetano R. Epidemiologia do uso de álcool no Brasil. Rev. Bras. Psiquiatr. 2004; 26(1):3-6.

16. Wilsnack SC, Wilsnack RW. Gender and alcohol: Individual and social perspectives. New Brunswick: Rutgers Center of Alcohol Studies; 1997.

17. Kerr-Correa F, Tucci AM, Hegedeus AM, Trinca LA, Oliveira JB, Floripes TMF, Kerr LRFS. Drinking patterns between men and women in two distinct Brazilian communities. Rev. Bras. Psiquiatr. 2008; 30(3):235-242.

18. Richman JAT, Zlatoper KW, Zackula Ehmke JL, Rospenda KM. Retirement and drinking outcomes: Lingering effects of workplace stress? Addict Behav 2006; 31(5):767-776.

19. Pimenta FAP, Simil FF, Tôrres HOG, Amaral CFS, Rezende CF, Coelho TO, Rezende NA. Avaliação da qualidade de vida de aposentados com a utilização do questionário SF-36. Rev. Assoc. Med. Bras. 2008; 54(1):55-60.

20. Domingos JBC, Pillon SC. O uso de álcool entre motoristas no interior do estado de São Paulo. $R$ Enferm UERJ 2007; 15(3):393-399.

21. Mascarenhas MDM, Malta DC, Silva MMA, Carvalho CG, Monteiro RA, Marais-Neto OL. Consumo de álcool entre vítimas de acidentes e violências atendidas em serviços de emergência no Brasil, 2006 e 2007. Cien Saude Colet 2009; 14(5):1789-1796.

22. Laranjeira R, Pinsky I, Zaleski M, Caetano R. I Levantamento nacional sobre os padrões de consumo de álcool na população brasileira. [site da Internet]. [acessado 2013 out 13]. Disponível em: http:// www.obid.senad.gov.br

Artigo apresentado em 08/08/2012

Aprovado em 30/09/2012

Versão final apresentada em 26/10/2012 
